\title{
Starter formula enriched in prebiotics and probiotics ensures normal growth of infants and promotes gut health: a randomized clinical trial
}

\author{
Michael Radke', Jean-Charles Picaud ${ }^{2}$, Andrea Loui ${ }^{3}$, Gilles Cambonie ${ }^{4}$, Dirk Faas ${ }^{5}$, Harry N. Lafeber 6 , Nanda de Groot ${ }^{7}$, \\ Sophie S. Pecquet ${ }^{7}$, Philippe G. Steenhout ${ }^{8}$ and Jean-Michel Hascoet ${ }^{9}$
}

BACKGROUND: Prebiotics and probiotics exert beneficial effects by modulating gut microbiota and immune system. This study evaluates efficacy and safety of an infant formula containing bovine milk-derived oligosaccharides and Bifidobacterium animalis ssp lactis (B. lactis) (CNCM I-3446) on incidence of diarrhea and febrile infections during the first year of life (primary outcome).

METHODS: Full-term infants receiving Test or Control (without bovine milk-derived oligosaccharide and B. lactis) formulae were enrolled in a multicenter, randomized, controlled, and double-blind trial with a reference breastfeeding group. .

RESULTS: 413 infants were assigned between Test $(n=206)$ and Control $(n=207)$ formula. There was no significant difference for diarrhea and febrile infections incidence between groups at 6 (odds ratio ( $95 \%$ confidence interval) $=0.56(0.26-$ 1.15), $P=0.096)$ and 12 mo (odds ratio $=0.66(0.38-1.14$ ), $P=0.119$ ). Test formula was well tolerated, anthropometrics parameters were not significantly different between groups and aligned with WHO growth standards up to 12 mo. Data from test group showed that gut microbiota pattern, fecal IgA and stool pH were brought to be closer to those of breastfed infants.

CONCLUSION: An infant formula enriched with bovine milkderived oligosaccharide and B. lactis supports normal infant growth, is well tolerated and improves intestinal health markers. No differences in diarrhea and febrile infection incidence were found in the population studied.

B reastfed infants are better protected against infections than formula fed infants (1-3). This difference stems partly from the difference in the composition of the gut microbiota in these two populations. Breast milk stimulates the development of a bifidobacterium rich microbiome in full-term infants (4). Anaerobes such as Clostridium sp., Bacteroides sp., and facultative anaerobes such as Escherichia $s p$. and enterococci are isolated more rarely in breastfed infants than in formula fed infants $(5,6)$. By contrast, formula fed infants have a more diversified microbiota (4).

The gut microbiota is involved in the normal development of the intestinal mucosal immune system (7) and, in breastfed infants, the bifidobacterium-rich microbiota is thought to have an immunoprotective role. Furthermore, the bifidobacterium-rich microbiota creates an acidic environment, by producing metabolites that is considered to be hostile to pathogen growth (8).

The development of new formulas attempts to incorporate some of the beneficial functional properties of breast milk into formulas. This includes adding probiotic strains such as bifidobacterium and lactobacilli and undigestible oligosaccharides that selectively stimulate bifidobacterium growth. In addition to stimulating bifidobacterium and lactobacilli growth, prebiotics also modulate the immune system, which can contribute to protection against infections (9).

In this study we evaluated the effect of a Test formula containing bovine milk-derived oligosaccharide (BMOS) and the probiotic Bifidobacterium animalis ssp lactis (B. lactis) on the immune system. We have already evaluated the effects of this prebiotic-probiotic mix in infant formula on the gut microbiota in early life, showing modulation of gut microbiota closer to that of breastfed infants (10). The aim of this study was focused on microbiota modulation effect on immune maturation: comparing the rate of infectious diarrhea and other infections. Additionally, anthropometric measures, digestive tolerance, fecal $\mathrm{pH}$ and gut microbiome, behavioral measures, and immune markers were assessed in infants fed the Test formula compared with those fed a Control formula.

\section{METHODS}

\section{Study Design}

This was a multicenter, randomized, double-blind, and controlled study. It included two randomized arms (Test group fed formula with BMOS and B. lactis, and Control group fed identical formula without

\footnotetext{
${ }^{1}$ Klinik für Kinder und Jugendmedizin, Klinikum Westbrandenburg GmbH, Potsdam, Germany; ${ }^{2}$ Service de Réanimation Néonatale, Hôpital de la Croix Rousse, Lyon, France; ${ }^{3}$ Klinik für Neonatologie, Charité Campus Virchow-Klinikum, Berlin, Germany; ${ }^{4}$ Service de Pédiatrie 2, Hôpital Arnaud de Villeneuve, Montpellier, France; ${ }^{5}$ Zentrum für Kinderheilkunde \& Jugendmedizin, Universitätsklinikum Gießen, Germany; ${ }^{6}$ Afdeling Kindergeneeskunde, Vrije Universiteit Medisch Centrum, Amsterdam, The Netherlands; ${ }^{7}$ Research \& Development, Nestlé Nutrition, Vevey, Switzerland; ${ }^{8}$ Medical Nutrition, Nestle Health Science, Vevey, Switzerland; ${ }^{9}$ Maternité Régionale Universitaire A. Pinard, CHRU, Nancy, France. Correspondence: Jean-Michel Hascoet (j.hascoet@chru-nancy.fr) 
BMOS and B. lactis) and a reference group. Infants were enrolled from October 2008 to December 2013. The study was conducted in three sites in Germany (Klinik für Geburtsmedizin, Charité, Berlin; Zentrum für Kinderheilkunde und Jugendmedizin, Universitätsklinikum Gießen; and Klinikum Ernst von Bergmann, Potsdam); three sites in France (Service de réanimation néonatale, Hôpital de la croix rousse, Lyon; Service de pédiatrie 2, Arnaud de Villeneuve, Montpellier and; Service de Néonatologie, Maternité Régionale Universitaire, Nancy), and one site in Netherlands (Afdeling Kindergeneeskunde, Vrije Universiteit Medisch Centrum, Amsterdam). The Institutional Review Board/ Independent Ethics Committee of each site reviewed and approved the study protocol and informed consent form.

\section{Study Population}

Healthy full-term infants aged $0-14 \mathrm{~d}$ old whose mothers had elected not to breastfeed were enrolled in the formula groups. Exclusively breastfed infants whose mothers had chosen to breastfeed for at least the first 3 mo were enrolled in the reference group.

Infants participating in the study had to fulfill the following inclusion criteria: being full-term (between 37 and $42 \mathrm{wk}$ gestation); being $\leq 14 \mathrm{~d}$ old at the time of enrollment; weighing $\geq 2,500 \mathrm{~g}$ and $\leq 4,500 \mathrm{~g}$; and having their legal representative's informed consent. Infants were excluded from the study if they had congenital illness or malformation that could affect normal growth; significant prenatal or postnatal diseases; perinatal antibiotic intake; mothers who had an acute infection during the last month of pregnancy; rehospitalization for more than $2 \mathrm{~d}$ in the first $14 \mathrm{~d}$ of life (with the exception of hospitalization due to jaundice); receiving infant formula containing probiotics or prebiotics at the time of enrollment; parents/caregivers who were expected not to be able to comply with the study procedure; and participating in another interventional clinical trial.

\section{Study Formulas and Blinding}

Both Test and Control formulae contained sufficient amounts of protein $(1.8 \mathrm{~g} / 100 \mathrm{kcal}$, whey/casein ratio of $70: 30)$, carbohydrates, fats, vitamins, and minerals to support the normal growth of healthy infants from birth to 6 mo of age. The Test formula was additionally supplemented with BMOS: a mixture of BMOSs generated from whey permeate (containing galacto-oligosaccharides and milk oligosaccharides such as $3^{\prime}$ and $6^{\prime}$ - sialyllactose) at a total oligosaccharide concentration of $5.8 \pm 1.0 \mathrm{~g} / 100 \mathrm{~g}$ of powder formula $(8 \mathrm{~g} / \mathrm{l}$ in the reconstituted formula) and a probiotic B. lactis (CNCM I-3446, $1 \times 10^{7} \mathrm{cfu} / \mathrm{g}$ of powder formula).

All study subjects received a follow-up formula from 6-12 mo of age without pre- and probiotics. Introduction of complementary food progressively started between 4 and 6 mo of age. Formulae were manufactured at the Nestlé Product Technology Center (Konolfingen, Switzerland). The sponsor blinded the study formulas, which were distinguishable only by the letters printed on the tin label (one code per group) and the color of the labels. Parents (caregivers), investigators, study support staff, and the clinical project managers were blinded to the identity of the products.

\section{Outcomes Measures}

The primary efficacy outcome was the mean incidence of diarrhea and all infections with fever during the first 6 mo and $1 \mathrm{y}$ of life. Diarrhea was defined as three or more loose or watery stools in $24 \mathrm{~h}$. An episode of diarrhea was considered to have ended once there were two consecutive stools that were not watery or if no stool had been passed for $24 \mathrm{~h}$. Fever was defined body temperature $>38^{\circ} \mathrm{C}$ and reaching $38.5^{\circ} \mathrm{C}$ at least once during the last $24 \mathrm{~h}$.

Secondary outcomes were adverse events $(\mathrm{AE})$, daily weight gain during the first $4 \mathrm{mo}$, weight, length, head circumference, arm circumferences, triceps, and subscapular skinfold measurements, digestive tolerance and colics during the 12 -mo study period, and fecal $\mathrm{pH}$, fecal bacterial counts, fecal IgA and $\alpha-1$ antitrypsin concentration and salivary $\operatorname{IgA}$ concentrations at 3 and 6 mo.

\section{Study Conduct}

At enrollment, the infants' and their mothers' demographic data were recorded. Birth anthropometric measurements, 1-, 5-, and 10-min APGAR scores, and medical history of infants were recorded. Eligible infants were randomized between formula groups.
Parents/caregivers received 3-d diaries for recording of formula intake and digestive tolerance (stool characteristics, occurrence of flatulence, and colic) for the $3 \mathrm{~d}$ preceding each visit to the study center.

All infants visited the study site at $1 \mathrm{mo}( \pm 3 \mathrm{~d}), 2$ mo $( \pm 3 \mathrm{~d}), 3$ mo $( \pm 3 \mathrm{~d}), 6 \mathrm{mo}( \pm 7 \mathrm{~d}), 9 \mathrm{mo}( \pm 7 \mathrm{~d})$, and $12 \mathrm{mo}( \pm 7 \mathrm{~d})$ of age. At each visit, the investigator examined the infants, assessed any adverse events (AEs), took their anthropometric measurements, and reviewed their medical records and 3-d diaries. Saliva and fecal samples were collected from a subset of infants at 3 and $6 \mathrm{mo}$.

Assessments of AEs. The investigators assessed for all enrolled infants episodes of cough, fever, skin rash, and antibiotic intake occurring between visits based on interviews with parents/caregivers. Atopic eczema was recorded on a scale of $0-3$. The number of days of antibiotic intake was recorded. AEs were coded using the World Health Organization Adverse Drug Reaction Terminology (WHO ART).

Lower respiratory tract infections (rhinitis, pharyngitis, otitis, and common cold) were identified by at least one of the following: rales or crepitations, wheezing, stridor, respiratory rate $>50 / \mathrm{min}$, cyanosis, or chest in drawing (bronchiolitis, bronchitis, and pneumonia). Upper respiratory tract infection was defined as having at least one of the following: purulent runny nose, sore throat, cough, or earache or ear discharge. Both types of infections were recorded together as all respiratory tract infections.

Anthropometric Measurements. At each study site, infants were weighed nude to the nearest $10 \mathrm{~g}$ on the same electronic scales calibrated according to the manufacturer's instructions. Recumbent length was measured on a standardized length board to the nearest $1 \mathrm{~mm}$ with the body fully extended and feet flexed. Head circumference was measured using a standard plastic-coated measuring tape at $\sim 2.5 \mathrm{~cm}$ above the eyebrows.

Digestive Tolerance. Digestive tolerance was assessed based on the records in the 3-d diaries. These were mean 24-h stool frequencies; predominant consistency (hard, formed, soft, or loose/watery); Colics were recorded as present or absent, and were assessed in infants up to $3 \mathrm{mo}$ of age. They included paroxysms of irritability, fussing, or inconsolable crying that start and stop without obvious cause; episodes lasting $\geq 3 \mathrm{~h} / \mathrm{d}$ and occurring $\geq 3 \mathrm{~d} / \mathrm{wk}$ for $\geq 1 \mathrm{wk}$; but without failing to thrive.

Salivary IgA. Salivary IgA were measured with a specific enzymelinked immunosorbent assay kit by an immuno-automated analyzer (EVOLIS-BioRad, Marnes la Coquette, France). After extraction, the samples were frozen until their analysis.

Fecal pH and Bacterial Counts. Fecal samples were collected at home by parents. Approximately $10 \mathrm{~g}$ of fresh fecal samples were placed into a sterile tube within $\leq 30$ min of emission. Tubes were placed in an aluminum bag along with a packet of AnaeroGen (Oxoid, Pratteln, Switzerland). The tightly closed bags were kept at $4^{\circ} \mathrm{C}$ and transported on ice to the study site within $5-10 \mathrm{~h}$ after emission. At each study site, fecal $\mathrm{pH}$ was measured using the same $\mathrm{pH}$ meter (electrode from Metrohm, Zofingen, Switzerland).

For fecal bacterial analysis, $10 \%$ glycerol was added to $2 \mathrm{~g}$ of fecal sample and stored at $-80^{\circ} \mathrm{C}$. Fluorescent in situ hybridization (FISH) was performed by Biovisible (Groningen, The Netherlands), and analyses for the presence of $B$. lactis was performed by AAT (Fiorenzuola d'Arda, Italy).

Total protein, sIgA, and $\alpha-1$ antitrypsin concentrations measurements in fecal samples, $1 \mathrm{ml}$ of extraction buffer $(6 \mathrm{ml}$ EDTA of $0.5 \mathrm{~mol} / \mathrm{l}[\mathrm{pH} 7.6$ and $0.35 \mathrm{ml}$ of $30 \mathrm{mg} / \mathrm{ml}$ soybean trypsin inhibitor in a final volume of $100 \mathrm{ml}$ PBS) was added to each $0.5 \mathrm{~g}$ of stool sample and homogenized. Samples were vortexed for 1-2 min and centrifuged at $13,000 \mathrm{~g}$ for $15 \mathrm{~min}$ at $4^{\circ} \mathrm{C}$. Approximately $250 \mu \mathrm{l}$-aliquots of the supernatant were stored at $-20^{\circ} \mathrm{C}$. Total protein concentration was determined using the Pierce BCA Kit (Pierce, Ecublens, Switzerland). sIgA (Immundiagnostik, Bensheim, Germany) and $\alpha-1$ antitrypsin (Immunodiagnostik) concentrations were determined by immunoenzyme analyses.

Infants with any of the following were considered to have had major protocol violation: experiencing a life-threatening event during 


\section{Articles $\mid$ Radke tal.}

the study period, especially if it resulted in a change of regimen; hospitalization for more than $3 \mathrm{~d}$; introduction of complementary food before $4 \mathrm{mo}$; or nonexclusive feeding of assigned formulas during the study. Nonexclusive feeding was defined as intake of more than one bottle of another standard formula per week, being off study formula for more than three consecutive days, or intake of formula in the Breast-fed group.

\section{Randomization}

Infants were randomized into the formula groups using a computer software program which could be accessed via the internet software TrialBalance (Nestle Research Center, Vers-chez-les-Blanc, Switzerland). The strata for the randomization were delivery-type and gender. Subject id and the strata were entered into the program, the program returned the treatment code.

\section{Statistical Methods}

Sample size was calculated based on showing superiority of the Test formula in reducing the rate of diarrhea and overall infections during the first 6 and 12 mo of life. Based on previous studies $(11,12)$, differences $\geq 20 \%$ between the two groups were considered clinically significant. Significance level was set at $1.25 \%$ since two outcomes (diarrhea and overall infections) and two comparisons (at 6 and 12 mo) were made. Statistical power was $90 \%$. Based on these conditions, 155 infants per group had to complete the study to show significant differences between groups. Assuming a 25\% drop-out rate, 207 infants had to be enrolled in each formula group. For the reference breast-fed group, 63 infants were enrolled. Sample size calculation was performed using PASS 2005. This sample size was also adequate for demonstrating noninferiority in daily weight gain in the Test formula group compared with the Control formula group. The noninferiority margin was $3 \mathrm{~g} / \mathrm{d}$ and the estimated SD $6.1 \mathrm{~g} / \mathrm{d}$.

The intention to treat (ITT) populations included all randomized infants who had any intake of the study formulas and had attended
$\mathrm{V} 1$ at 1 mo of age. The PP population included all randomized infants with no major protocol violations

The primary outcome analysis, incidence rate of diarrhea and infections with fever, was analyzed in the ITT and PP populations and compared between groups using Fisher's Exact-test. Significance was set at $P=0.0125$ since there were four primary comparisons. Andersen-Gill modeling approach was also used to compare diarrhea rates between Test and Control groups as well as between the Test and Breastfed groups over the 12-mo study period in ITT population. The analysis of diarrhea rates included all reported diarrhea incidents that likely encompassed infectious diarrhea and perceived diarrhea (e.g., watery stool); therefore diarrhea + associated symptoms were used as a surrogate for identifying infectious diarrhea and Fisher's Exact tests were conducted to compare incidence rates of infectious diarrhea over the $12 \mathrm{mo}$ in the Test and Control groups.

Secondary outcomes were analyzed in the ITT population and no adjustments for multiplicity were performed. Daily weight gain for each infant was calculated by subtracting the baseline weight from the weight at each visit then dividing this difference by the age of the infant in days. Daily weight gain was compared between groups using ANOVA, and the Test was considered to be noninferior to the Control if the lower limit of the one-sided 95\% CI was $>3 \mathrm{~g} / \mathrm{d}$. Other anthropometric measurements were also analyzed by ANOVA. Additionally, weight, length, and head circumference were compared with WHO standards (13).

For normally distributed continuous variables (i.e., weight, length, head circumference, and BMI), the following linear mixed models were constructed to compare Test and Control; and Test and breastfed groups. Baseline measures of corresponding dependent variables, gender, and delivery mode were included in the model: dependent variable (e.g., weight $)=$ baseline + group $^{*}$ visit + gender + delivery + random effects (within-subject variability). If logarithmic transformation was needed to ensure normal distribution of data, the logtransformed data were used in the analysis.

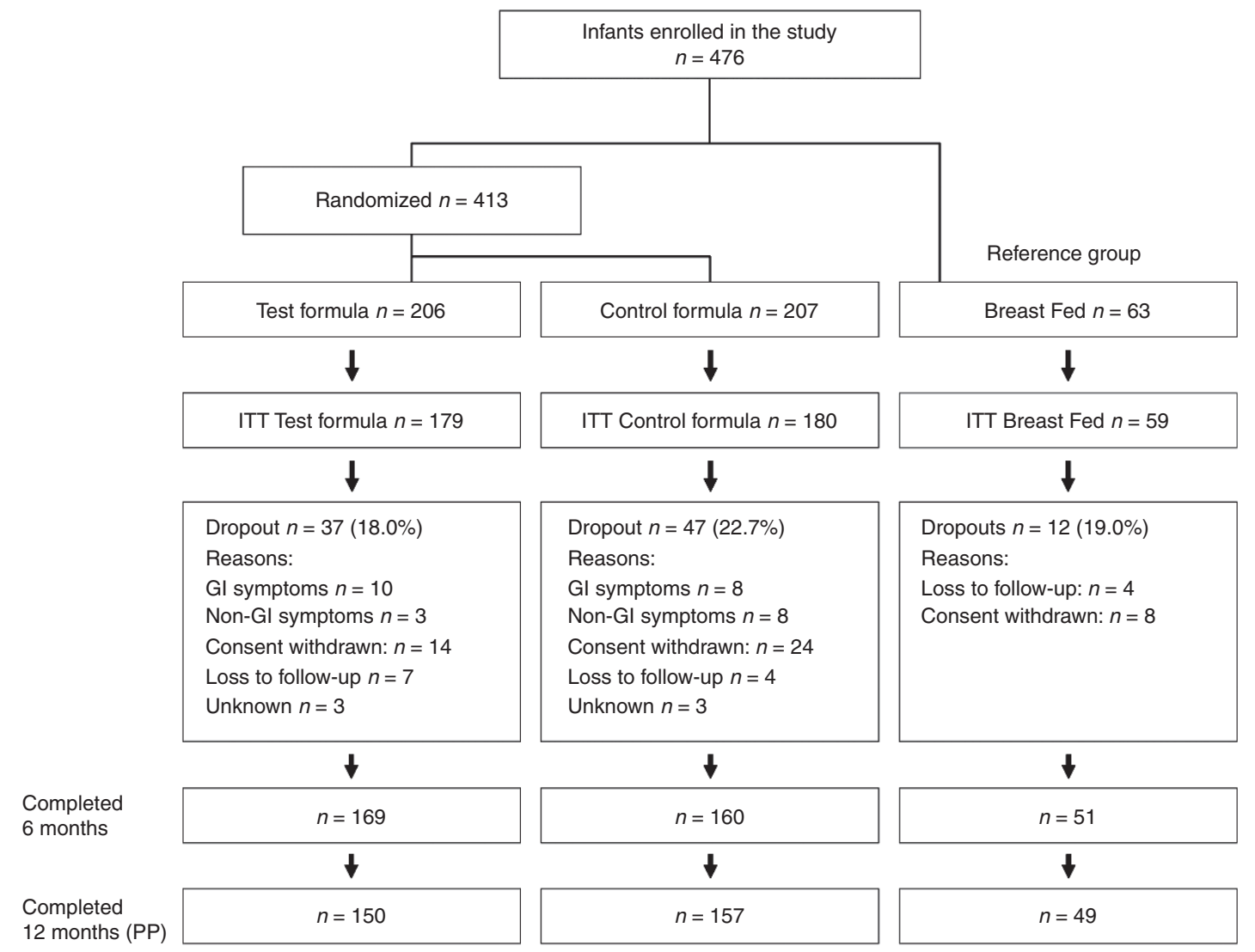

Figure 1. Flow chart of study. 
For secondary endpoints, normally distributed continuous variables without baseline values (i.e., z-scores, average formula intake per day, stool $\mathrm{pH}$, bacteriology tests, saliva and stool sIgA concentrations, and stool $\alpha-1$ antitrypsin concentration), similar linear mixed models but without the baseline parameter were constructed to compare Test and Control; and Test and Breastfed groups.

Continuous variables that were not normally distributed (even after log-transformation, i.e., daily number of stool and number of colics) were analyzed at each time point using nonparametric tests (i.e., Wilcoxon rank-sum tests). Categorical variables (i.e., morbidity frequency and stool consistency,) were analyzed at each time points using Fisher's exact tests.

Serious and nonserious AEs were assessed in all enrolled infants, including those that had no intake of the study formulas. All analyses were performed using the statistical software R version 3.01.

\section{RESULTS}

\section{Study Population and Baseline Characteristics}

About 476 infants were enrolled, with 206 randomized into the Test formula group and 207 in the Control formula group. Sixty-three infants were enrolled in the breast-fed group. A total of 58 infants (27 in each of the Test and the Control groups and four in the Breast-fed group) were excluded from the ITT analyses because they dropped out before the 1-mo visit. The population that completed the entire study duration was 150 infants in the Test group, 157 in the Control group, and 49 in

Table 1. Demographics and baseline characteristics of infants and their mothers

\begin{tabular}{|c|c|c|c|}
\hline & $\begin{array}{l}\text { Test formula } \\
n=206\end{array}$ & $\begin{array}{c}\text { Control formula } \\
\quad n=207\end{array}$ & $\begin{array}{c}\text { Breast-fed } \\
n=63\end{array}$ \\
\hline \multicolumn{4}{|l|}{ Infants' Characteristics } \\
\hline Cesarean delivery, $n(\%)$ & $37(18)$ & $37(18)$ & $10(16)$ \\
\hline Female, $n(\%)$ & $103(50)$ & $107(52)$ & $30(48)$ \\
\hline Antibiotherapy, $n$ (\%) & $0(0)$ & $1(0)$ & $0(0)$ \\
\hline Weight, median (IQR) ${ }^{\mathrm{a}} \mathrm{kg}$ & $3.3(3.0,3.6)$ & $3.3(3.0,3.6)$ & $3.3(3.1,3.7)$ \\
\hline Length, median (IQR) cm & $50(48,51)$ & $49(48,51)$ & $51(49,51)$ \\
\hline $\mathrm{BMI}^{\mathrm{b}}$, median $(\mathrm{IQR}) \mathrm{kg} / \mathrm{m}^{2}$ & $13.6(12.8,14.2)$ & $13.5(12.7,14.4)$ & $13.3(12.6,13.8)$ \\
\hline $\mathrm{HC}^{c}$, median (IQR) cm & $35.0(34.0,35.5)$ & $34.0(33.5,35.0)$ & $35.0(34.0,35.5)$ \\
\hline \multicolumn{4}{|l|}{ Mothers' Characteristics } \\
\hline $\begin{array}{l}\text { Smoking during } \\
\text { pregnancy, } n(\%)\end{array}$ & $55(27)$ & $65(32)$ & $3(5)$ \\
\hline $\begin{array}{l}\text { Pregnancy alcohol } \\
\text { intake, } n(\%)\end{array}$ & $12(6)$ & $12(6)$ & $2(3)$ \\
\hline Age, median (IQR) y & $29(25,32)$ & $28(24,32)$ & $31(28,34)$ \\
\hline $\begin{array}{l}\text { Years of education, } \\
\text { median (IQR) }\end{array}$ & $7(5,10)$ & $7(5,10)$ & $12(10,13)$ \\
\hline
\end{tabular}

${ }^{\mathrm{a}} \mathrm{QR}$, inter quartile range. ${ }^{\mathrm{b}} \mathrm{BMI}$, body mass index. ${ }^{\mathrm{c}} \mathrm{HC}$, head circumference. the Breastfed group. (Figure 1). Demographic and baseline characteristics were balanced between formula groups (Table 1). Mothers of breastfed infants appeared to have higher education and fewer of them smoked during pregnancy (Table 1).

\section{Incidence of Diarrhea and Infections with Fever}

The number of infants who had diarrhea and infections with fever at 6 and 12 mo was not significantly different between the formula groups (Table 2). Analysis of the PP population gave similar results (data not shown). Similar proportions of infants in the Test and Control formula groups had diarrhea (Table 2). The odds ratio of diarrhea in the Test vs. Control group was 0.56 (95\% confidence interval (CI): 0.26 to 1.15$), P=0.096$ at 6 mo and 0.66 (95\% CI: 0.38 to 1.14 ), $P=0.119$ at 12 mo.

The Andersen-Gill modeling approach was employed to compare diarrhea incidence rates between all groups, Test vs. Control and Test vs. Breastfed groups. Figure 2 shows the Kaplan-Meier plot in which the transition from being without diarrhea to having diarrhea incidence throughout $12 \mathrm{mo}$ is indicated by using the incidence rate calculated from number of infants with at least one incident or no incident in the ITT population. It shows that the diarrhea incidence was not significantly different between Test group and Control group, and between Test and Breastfed group (hazard ratio Test vs. Control group 0.56 (95\% CI: 0.338 to -0.910$), P=0.0252$ and Test vs. Breastfed group 1.12 (95\% CI: 0.601 to 2.102 ), $P=$ 0.7205; Figure 2).

The proportion of infants with AEs related to infections was comparable between the formula groups (Table 3). Between 65 and $74 \%$ of infants in the formula and Breast-fed groups had $\geq 1$ AE. In about $2-5 \%$ of these, the AEs were considered to be related to the formulas or breast feeding. Seventy-seven specific AEs were reported in 48 infants. The most common specific AEs were in the infections and infestations system organ class: 21 in the Test group, 16 in the Control group, and 3 in the Breastfed group.

\section{Growth and Body Composition Measurements}

Mean daily weight gain during the first 6 mo of life was not significantly different between infants in the Test and Control formula groups (difference between the two groups at 6 mo was $-0.715 \mathrm{~g} / \mathrm{d}$ (95\% CI: -1.6 to 0.01$)$, ITT). The lower limit of the $95 \%$ CI of the difference in weight gain was above $-3 \mathrm{~g} / \mathrm{d}$, fulfilling the conditions for noninferiority of the Test to the Control with respect to growth.

Mean weight, length, BMI and head circumference-forage $\mathrm{z}$-scores throughout the 12 -mo study period were not

Table 2. Number of infants (\%) who had $\geq 1$ diarrhea episode or any infections with fever, intention to treat (ITT)

\begin{tabular}{|c|c|c|c|c|}
\hline Outcomes & Test formula $n=179$ & Control formula $n=180$ & Breastfed $n=59$ & $\begin{array}{l}P \text {-value (test } \\
\text { vs. control) }\end{array}$ \\
\hline Diarrhea from enrollment to $6 \mathrm{mo}$ & 25 (13.9) & $15(8.3)$ & $5(8.4)$ & 0.096 \\
\hline All infections from enrollment to $6 \mathrm{mo}$ & $38(21.2)$ & $38(21.1)$ & $10(16.9)$ & 1 \\
\hline All infections from enrollment to $12 \mathrm{mo}$ & $81(45.2)$ & $80(44.4)$ & $27(45.7)$ & 1 \\
\hline
\end{tabular}




\section{Articles | Radke et al.}

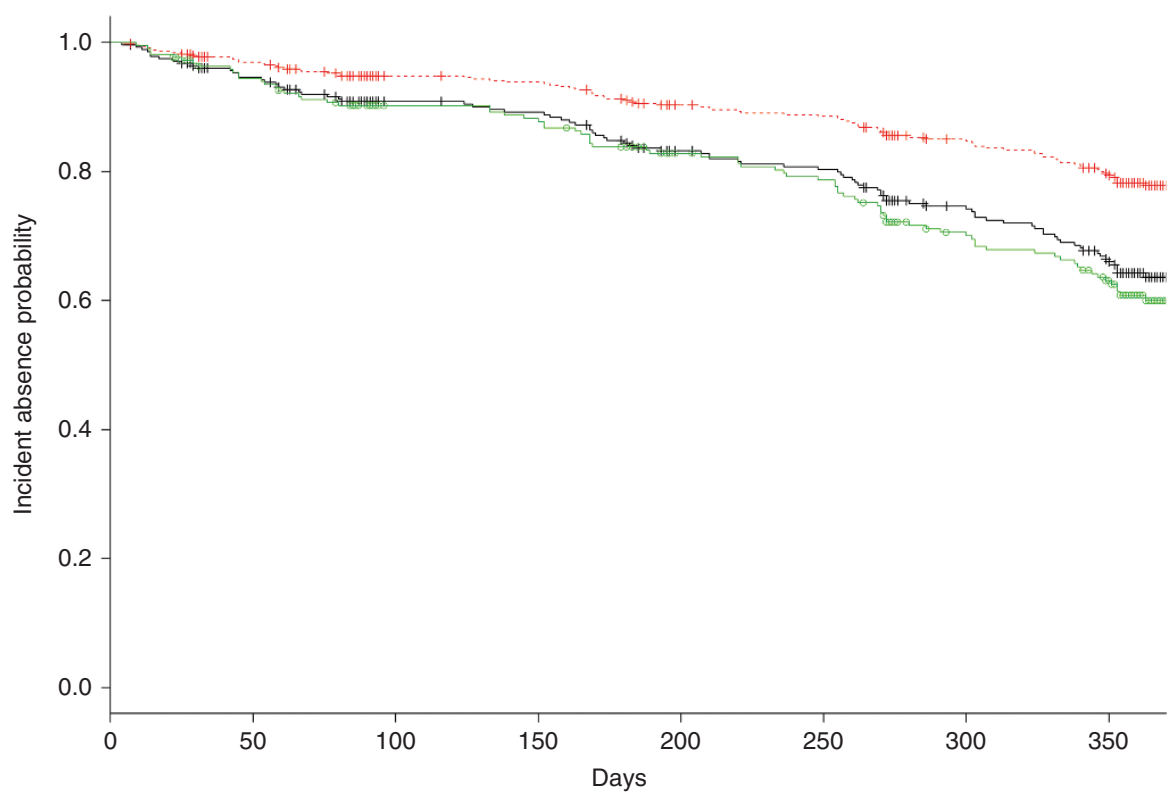

Figure 2. Kaplan-Meier plot for diarrhea incidence. Black line represents Test, red line represents Control, and green line represents breastfed. $(P=0.0252$ Test vs. Control, $P=0.7205$ Test vs. Breastfed)

Table 3. Number of infants (\%) with specific adverse events, intention to treat (ITT)

\begin{tabular}{lccc}
\hline SOC & $\begin{array}{c}\text { Test formula } \\
n=179\end{array}$ & $\begin{array}{c}\text { Control } \\
\text { formula } n=180\end{array}$ & $\begin{array}{c}\text { Breastfed } \\
n=59\end{array}$ \\
\hline $\begin{array}{l}\text { Respiratory, thoracic and } \\
\text { mediastinal disorders }\end{array}$ & $17(9.4)$ & $12(6.7)$ & $2(3.4)$ \\
$\begin{array}{l}\text { Gastrointestinal disorders } \\
\begin{array}{l}\text { Skin and subcutaneous } \\
\text { tissue disorders }\end{array}\end{array}$ & $24(41.1)$ & $84(46.9)$ & $31(52.5)$ \\
\hline
\end{tabular}

aSOC, system organ class according to World Health Organization adverse drug reaction terminology.

significantly different between the Test and Control formula groups (Figure 3). These growth measurements were also not significantly different between the Test and Breastfed groups.

\section{Digestive Tolerance}

The Test group tended to have less daily intake of formula than the Control group. The first month of life showed a difference of around $30 \mathrm{ml} / \mathrm{d}(P=0.08)$.

During the first 3 mo, the Test group had higher daily stool frequency compared with the Control group (median of 1.41.7 stools/d in the Test and 1.0- 1.5 stools/d in the Control, $P$ $<0.005$ for comparisons at each month). The stool frequency in the Test group was closer to the frequency observed in the Breastfed group (median frequency of 1.5-2.6 stools/d). From $6 \mathrm{mo}$, stool frequency did not vary significantly between the formula groups $(P>0.05)$.

For the first $3 \mathrm{mo}$, the proportion of hard and formed stools was higher among the Control formula group infants compared with the Test group the Test group profile being closer to that of the breastfed infants. The proportion of liquid stools showed to be higher among the Test group infants, as for the breastfed group, compared with the Control formula group. In the Breastfed group the proportion of liquid stools was the highest compared with soft and formed stools (Figure 4).

The occurrence of flatulence did not differ significantly between the two formula groups during the 12-mo study period. However, at 3 mo the proportion of infant who never had flatulence was higher in the Test group than in the control group ( 35 vs. $14 \%, P<0.01$ ). A lower proportion of infants in the Test group were reported to sometimes have flatulence (54 vs. $78 \%$ ). The incidence of colic did not differ significantly between formula groups.

\section{Fecal pH and Bacterial Counts}

Fecal $\mathrm{pH}$ was significantly lower in the Test group than in the Control group at both 3-mo (Test vs. Control): 5.74 (0.96) vs. 6.46 (0.65), $P=0.000$, and 6-mo: 6.03 (0.78) vs. $6.53(0.87), P=$ 0.000 . The $\mathrm{pH}$ level of the Test group was comparable to that of breastfed infants (Test vs. Breastfed: 5.74 (096) vs. 5.48 (0.73) at $3 \mathrm{mo}$ and $6.03(0.78)$ vs. 5.79 (0.83), Figure 5)

Stool bacteriological counts were examined in a subpopulation of the infants. At $3 \mathrm{mo}$, bifidobacterium and lactobacilli counts were significantly higher and clostridia/eubacteria counts were significantly lower in the Test group compared with the Control group $(P<0.01$, Figure 6a). Bifidobacterium counts were also higher in the Test group than in the Breastfed group, additionally Staphylococcus counts were lower in the Test (Figure 6a). All these differences were still observable at $6 \mathrm{mo}$ (Figure 6b).

At 3 mo, B. lactis was detected in $88.1 \%$ of stool samples in the Test group, as compared with $10.1 \%$ in the control group $(P=0.00)$. Similarly at $6 \mathrm{mo}, 84.6 \%$ of stool samples from the Test group, as compared with $12.2 \%$ in the Control group $(P=$ 0.00 ) were positive for $B$. lactis. 


\section{Immune Measurements}

Stool sIgA concentrations were significantly higher in the Test group compared with the Control group at both 3 and 6 mo $(P<0.0001$ for both time points, Table 4$)$. Stool sIgA concentrations in Test group vs. Breastfed group were not significantly different at 3 mo, but at 6 mo the sIgA levels were significantly lower compared with the Breastfed group $(P=0.0002$, Table 4$)$.

Salivary IgA concentrations were not significantly different between the formula groups at any time $(P>0.05)$. Stool $\alpha-1$ antitrypsin concentration was significantly higher in the Test group at $3 \mathrm{mo}(P=0.03)$ but not at $6 \mathrm{mo}(P=0.26)$. Compared with the Breastfed group, $\alpha-1$ antitrypsin was lower in the Test group, but the difference was significant only at 6 mo $(P<$ 0.001), (data not shown).

\section{DISCUSSION}

We investigated whether exclusive intake of a formula containing BMOS and B. lactis for 6 mo would reduce diarrhea and other infections with fever in infants during the first 6 mo or up to $1 \mathrm{y}$ of life. The proportion of infants who had diarrhea or infections with fever during the 6- and 12-mo periods of the study was not significantly different between the Test and Control formula groups or Test and Breastfed group.

The total number of all infants who had diarrhea over the study period was much lower than estimated based on previous studies. Whereas $<15 \%$ of infants at 6 mo and $<25 \%$ of infants at 12 mo had diarrhea, our study design was based on a $46 \%$ prevalence of diarrhea based on Chouraqui (11) and Arslanoglu (12). Thus, the study was underpowered (36\% power) to detect a difference between the groups: instead of the estimated sample size of 155 per group, 555 infants per group would have had to be assessed in order to be able to see a significant effect, meaning $80 \%$ power. Similarly, the study was likely to have been underpowered to detect a difference in the rate of infections with fever, though the power calculation was not based on this variable.
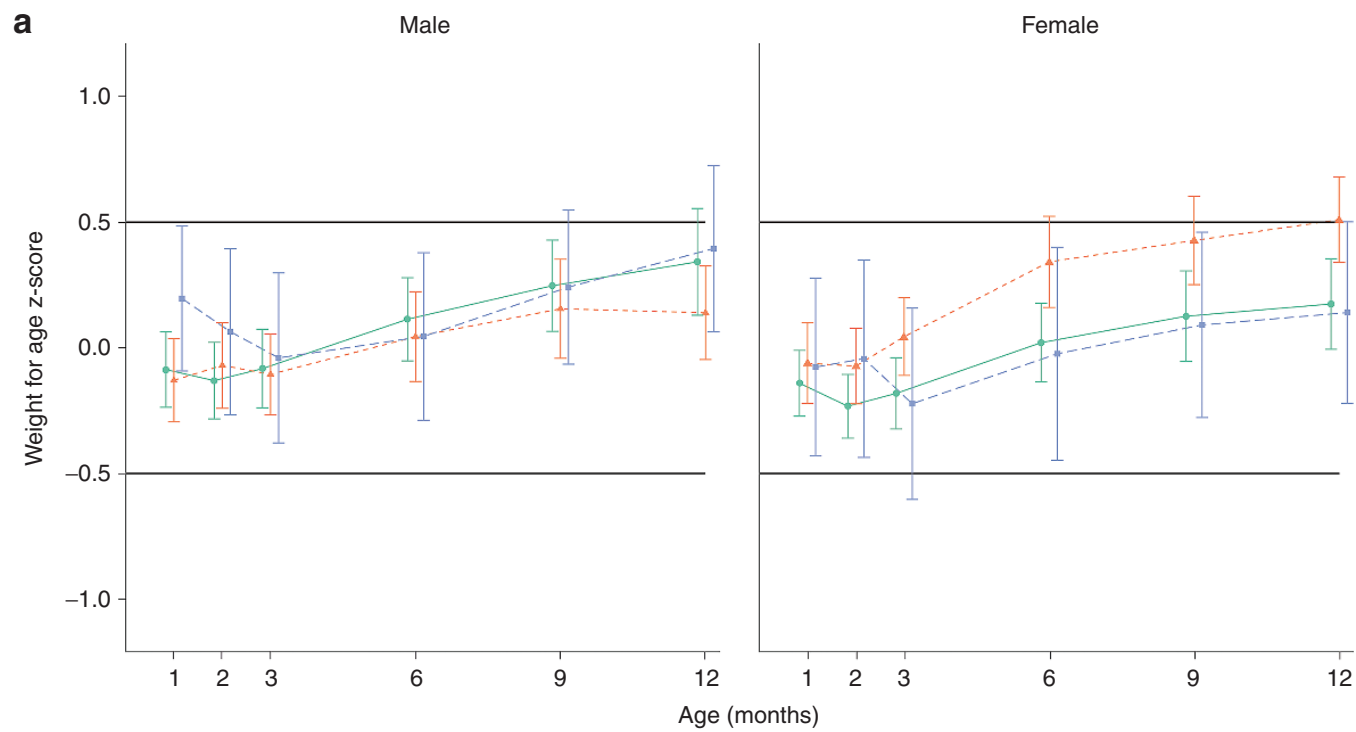

b
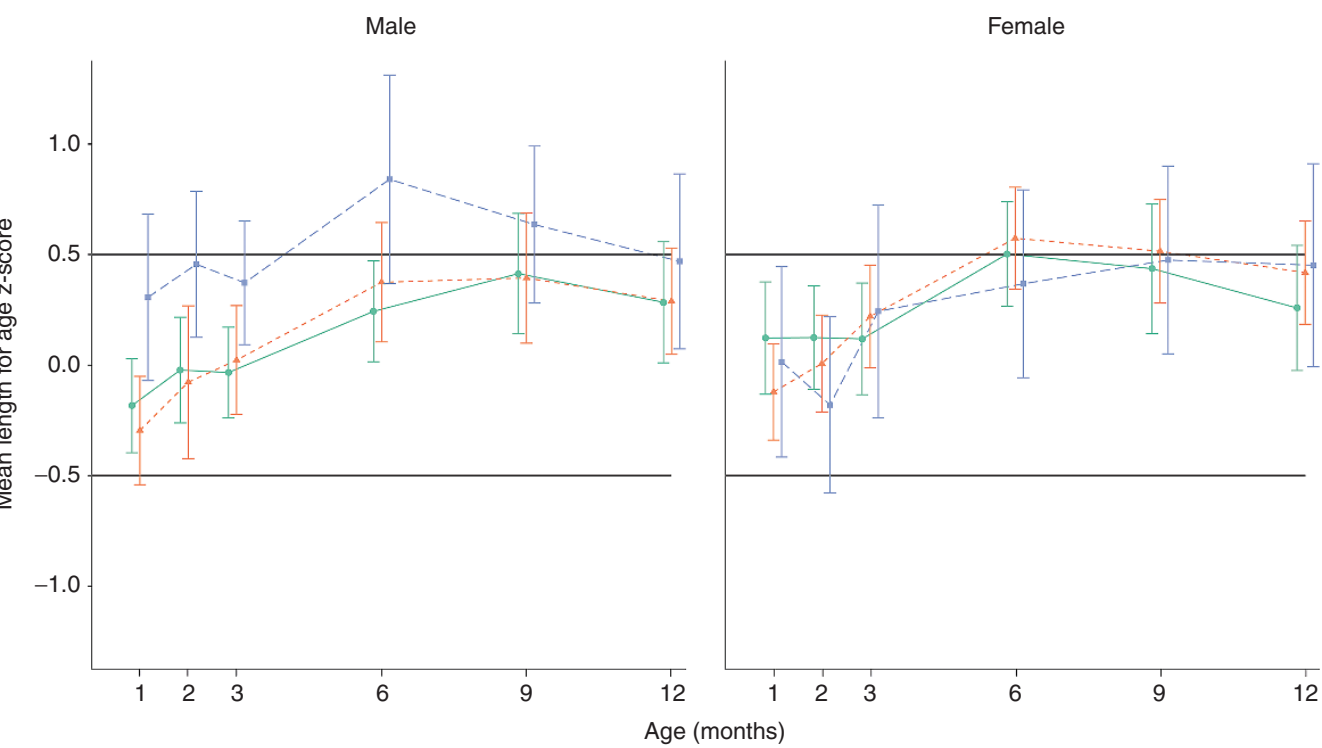


\section{Articles | Radke et al.}


Figure 3. Mean growth of infants based on WHO growth standards. (a) weight, (b) length, (c) head circumference, (d) body mass index for age-z-scores. Green lines represent Test, red lines Control, and blue lines Breastfed.

Among infants with diarrhea during the first year (43 in the Test and 31 in the Control groups), the presence of Escherichia coli, Campylobacter, and rotavirus was tested in 11, but none of these species was detected (data not shown). Furthermore, only 49 (26 in the Test and 23 in the Control groups) of the diarrhea cases were associated with other symptoms (e.g., fever), and only two infants were prescribed antibiotics. Other diarrheal agents could have been present in the stools, but in the absence of other symptoms it is unlikely that they were the causative agents. These findings suggest that very few of the diarrhea cases were infectious.

Additionally the Kaplan-Meier plot of the Test group, recording all diarrhea incidences, closely resembled that of the breastfed group, and both were seemingly different from that of the Control group (Figure 2). From the result it appears that the Control group had a lower incidence rate of diarrhea incidence compared with the Test group, likely because consuming the higher fiber content formula.

Test Formula gave more liquid stool consistency as for the Breastfed group, (Figure 4) which may have been perceived as diarrhea by parents/caregivers, according to the study protocol diarrhea definition.

The Test formula was well tolerated and supported normal growth in comparison with the reference breastfed group and the WHO growth standards. Even though during the first month infants in the Test group tended to consume less formula than those in the control group, this did not delay growth during this rapid growth phase. This suggests that BMOS and $B$. lactis may aid efficient absorption and utilization of nutrients. Scholz-Ahrens and colleagues have reported that bifidobacterium and lactobacilli improve absorption of various nutrients, minerals, and vitamins by the host (14). In our study, 


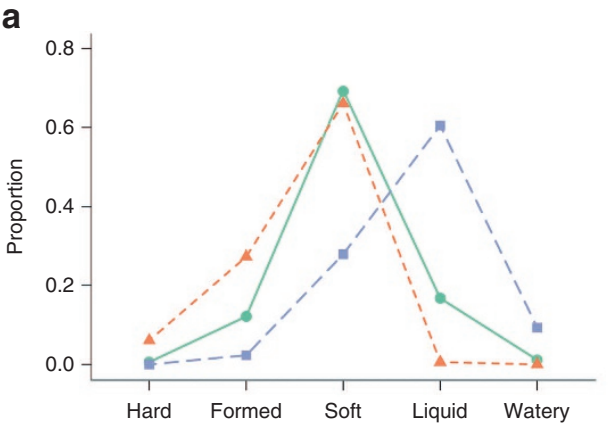

b

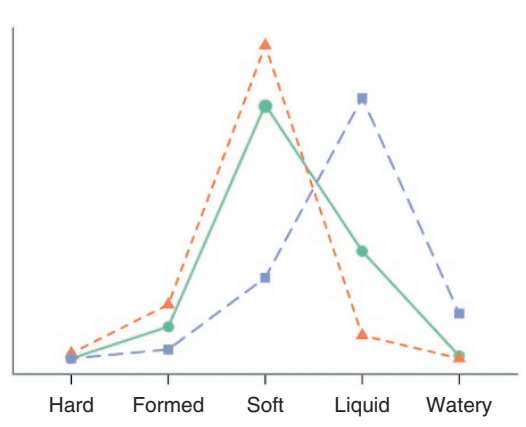

e

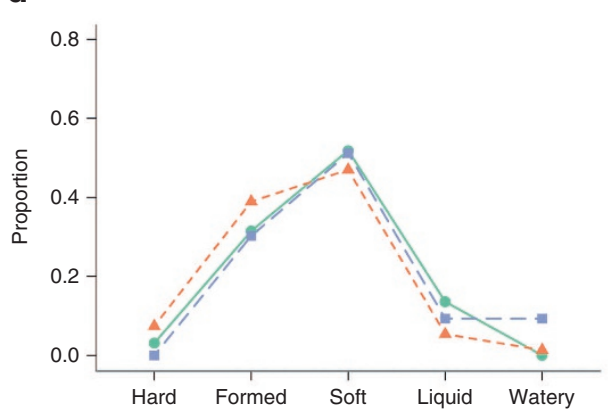

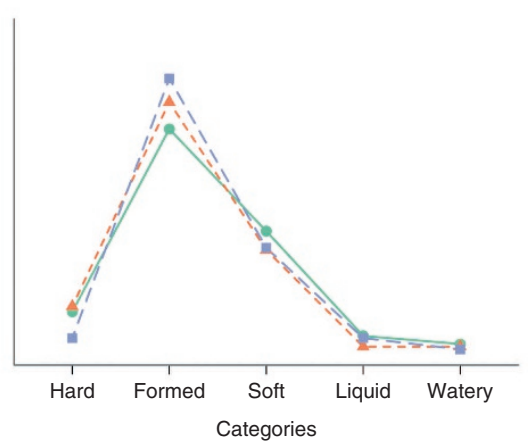

C

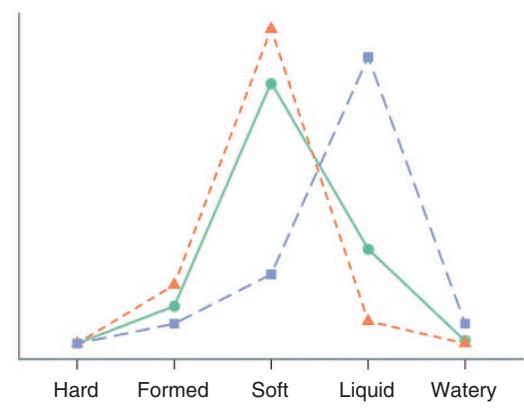

f

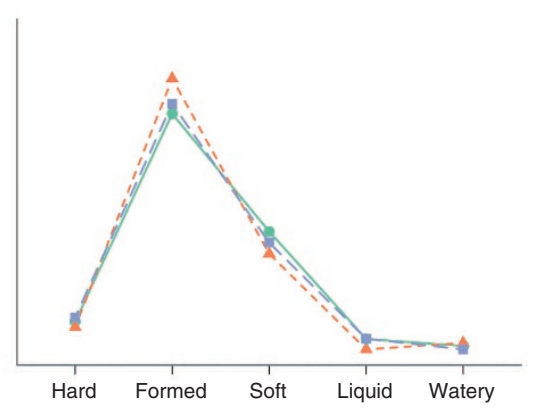

Figure 4. Stool consistencies at (a) 1, (b) $2,(\mathbf{c}) 3,(\mathbf{d}) 6,(\mathbf{e}) 9,(\mathbf{f}) 12$ mo. Green lines represent Test, red lines representControl, and blue lines represent Breastfed.



Figure 5. Stool $\mathrm{pH}$ at 3 and $6 \mathrm{mo}$. Circles are for Control, squares for Test and triangles for Breastfed. ${ }^{*} P=0.000,{ }^{*} P=0.078$, and ${ }^{\S} P=0.083$.

bifidobacterium and lactobacilli population were higher in the Test group (compared with Control), consistent with this idea. Additionally, the fermentation products of the nondigestible oligosaccharides, short chain fatty acids, can also be used by the host as a source of energy (15), which may have contributed to the growth of infants in the Test group.

In addition to the higher bifidobacterium and lactobacilli counts in the Test group, clostridia/eubacteria counts and fecal $\mathrm{pH}$ were lower in this group compared with the Control group. This suggests that the supplementation with BMOS and B. lactis promotes the development of potentially beneficial bacteria driving the gut ecosystem toward the one observed in breastfed infants. It is also of interest that Staphylococcus counts were lowered in the Test group. These findings are similar to those recently reported, where infants fed formula containing BMOS, B. longum and Lactobacillus rhamnosus or BMOS and $B$. lactis were shown to have higher bifidobacteria and lactobacilli counts and lower clostridia counts compared with a Control formula $(10,16)$.

The effect of formula containing BMOS and probiotic on gut microbiota composition and fecal $\mathrm{pH}$ was more profound than that observed in previous studies testing probiotics alone $(17,18)$. Holscher et al., reported that consuming B. lactis-supplemented formula (vs. Control formula) did not lower fecal $\mathrm{pH}$. These findings suggested that BMOS may play a key role in modifying the gut microbiota profile and fecal $\mathrm{pH}(10)$.

During the first months of life, regardless of feeding mode, the immune system matures, and sIgA are produced. Due to the additional contribution of sIgA from breast milk, the level of stool sIgA is naturally higher in the breastfed infants compared with formula fed infants. In this study, the intake of the Test formula significantly increased fecal sIgA at 3 and 6 mo compared with the Control formula, even reaching the levels observed in breastfed infant at 3 mo. Similar findings were made for $\alpha-1$ antitrypsin.

Increase in fecal sIgA and $\alpha-1$ antitrypsin, may contribute to enhanced mucosal resistance against gastrointestinal infections. A previous study (18) on probiotics showed that consuming B. lactis-supplemented formula increased fecal sIgA in vaginally delivered infants after $6 \mathrm{wk}$ of study formula consumption. Although direct comparisons of results between this study and the Holscher study were not strictly possible, the effects of BMOS and B. lactis supplementation on fecal 


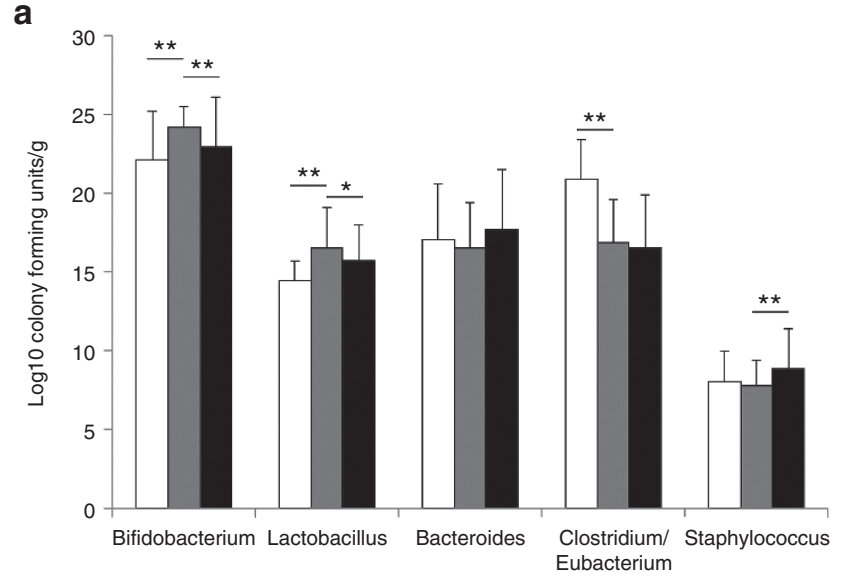

b



Figure 6. Bacterial counts at $3 \mathrm{mo}$ (panel a) and $6 \mathrm{mo}$ (panel b). White bars represent control, gray bars represent test and black bars represent Breastfed. ${ }^{*} P<0.05 ;{ }^{* *} P<0.01$.

Table 4. Mean (SD) concentration of immune markers

\begin{tabular}{lccc}
\hline $\begin{array}{l}\text { Immune } \\
\text { markers }\end{array}$ & Test formula & Control formula & Breastfed \\
\hline $\begin{array}{l}\text { Stool slgA (mg/l) } \\
3 \text { mo }\end{array}$ & $57.2(36.8)^{* *}, n=96$ & $33.8(32.7), n=77$ & $74.2(40.5), n=25$ \\
6 mo & $31.2(24.3)^{* *+}, n=96$ & $21.3(25.9), n=84$ & $53.7(32.9), n=35$ \\
Saliva slgA (mg/l) & & & \\
3 mo & $7.9(8.8), n=88$ & $7.1(5.3), n=73$ & $6.6(5.9), n=33$ \\
6 mo & $6.7(6.5), n=76$ & $7.1(6.6), n=72$ & $8.5(15.1), n=31$ \\
\hline${ }^{*} P<0.0001:$ comparison test with Control. & & \\
${ }^{\dagger} P=0.0002:$ comparison test with Breast-fed. & &
\end{tabular}

sIgA appear to be stronger than supplementation with B. lactis alone. This difference therefore suggests that BMOS plays a role in contributing to the higher stool sIgA and the immune modulation of the gut, most likely via microbiota modulation early in life.

Overall AEs were not significantly different between the formula groups. Most AEs were not considered to be related to the formulae, and the distribution of the ones that were considered related to study formula were similar between the groups.
In conclusion, although supplementation of infant formulas with BMOS and B. lactis did not show a decrease in diarrhea rates because of the overall low prevalence of diarrhea in the studied population, we show that it promotes a beneficial microbiota composition, increase in bifidobacteria and lactobacilli and decreases in clostridia/eubacteria counts, as observed in breastfed infants. It also helps a decrease in fecal $\mathrm{pH}$ and an increase fecal sIgA and $\alpha-1$ antitrypsin concentrations, suggesting favorable effect on immune markers and the gut milieu.

\section{ACKNOWLEDGMENTS}

The authors thank Claire Hubert for her commitment, and the diverse study teams for their major contributions to this study. They also acknowledge Antonio de Castro for the statistical analysis, Jian Yan for his scientific expertise and Annemarie Beekman for study co-ordination and follow-up. Finally, the authors are grateful to the families and caregivers who consented to their infants' participation in this study.

\section{STATEMENT OF FINANCIAL SUPPORT}

N.de G., S.S.P., and P.G.S. are Nestlé employees. The study was sponsored by Nestec Ltd.

Clinical trial registration: clinicaltrials.gov NCT01880970

Disclosure: There is no conflict of interest to disclose.

\section{REFERENCES}

1. Howie PW, Forsyth JS, Ogston SA, Clark A, Florey CD. Protective effect of breast feeding against infection. BMJ 1990;300:11-6.

2. Mountzouris KC, McCartney AL, Gibson GR. Intestinal microflora of human infants and current trends for its nutritional modulation. Br J Nutr 2002;87:405-20.

3. Quigley MA, Cumberland P, Cowden JM, Rodrigues LC. How protective is breast feeding against diarrhoeal disease in infants in 1990s England? A case-control study. Arch Dis Child 2006;91:245-50.

4. Rinne MM, Gueimonde M, Kalliomäki M, Hoppu U, Salminen SJ, Isolauri E. Similar bifidogenic effects of prebiotic-supplemented partially hydrolyzed infant formula and breastfeeding on infant gut microbiota. FEMS Immunol Med Microbiol 2005;43:59-65.

5. Collins MD, Gibson GR. Probiotics, prebiotics, and synbiotics: approaches for modulating the microbial ecology of the gut. Am J Clin Nutr 1999;69:1052S-7S.

6. Schrezenmeir J, de Vrese M. Probiotics, prebiotics, and synbioticsapproaching a definition. Am J Clin Nutr 2001;73(2 Suppl):361S-4S.

7. Walker WA. Initial intestinal colonization in the human infant and immune homeostasis. Ann Nutr Metab 2013;63 Suppl 2:8-15.

8. Sherman P, Cabana M, Gibson G, et al. Potential roles and clinical utility of prebiotics in newborns, infants, and children: proceedings from a global prebiotic summit meeting, New York City, June 27-28, 2008. J Pediatr 2009;155:S61-70.

9. Roberfroid M, Gibson GR, Hoyles L, et al. Prebiotic effects: metabolic and health benefits. Br J Nutr 2010;104 Suppl 2:S1-63.

10. Simeoni U, Berger B, Junick J, et al.; Study Team. Gut microbiota analysis reveals a marked shift to bifidobacteria by a starter infant formula containing a synbiotic of bovine milk-derived oligosaccharides and Bifidobacterium animalis subsp. lactis CNCM I-3446. Environ Microbiol 2016;18:2185-95.

11. Chouraqui JP, Grathwohl D, Labaune JM, et al. Assessment of the safety, tolerance, and protective effect against diarrhea of infant formulas containing mixtures of probiotics or probiotics and prebiotics in a randomized controlled trial. Am J Clin Nutr 2008;87:1365-73.

12. Arslanoglu S, Moro GE, Boehm G. Early supplementation of prebiotic oligosaccharides protects formula-fed infants against infections during the first 6 months of life. J Nutr 2007;137:2420-4.

13. WHO Multicenter Growth Reference Study Group. WHO child growth standards, 2006 (http://www.who.int/childgrowth/standards/en/). 
14. Scholz-Ahrens KE, Schrezenmeir J. Inulin and oligofructose and mineral metabolism: the evidence from animal trials. J Nutr 2007;137(11 Suppl):2513S-23S.

15. den Besten G, van Eunen K, Groen AK, Venema K, Reijngoud DJ, Bakker BM. The role of short-chain fatty acids in the interplay between diet, gut microbiota, and host energy metabolism. J Lipid Res 2013;54:2325-40.

16. Meli F, Puccio G, Cajozzo C, et al. Growth and safety evaluation of infant formulae containing oligosaccharides derived from bovine milk: a randomized, double-blind, noninferiority trial. BMC Pediatr 2014;14:306.

17. Langhendries JP, Detry J, Van Hees J, et al. Effect of a fermented infant formula scontaining viable bifidobacteria on the fecal flora composition and $\mathrm{pH}$ of healthy full-term infants. J Pediatr Gastroenterol Nutr 1995;21:177-81.

18. Holscher HD, Czerkies LA, Cekola P, et al. Bifidobacterium lactis Bb12 enhances intestinal antibody response in formula-fed infants: a random- ized, double-blind, controlled trial. JPEN J Parenter Enteral Nutr 2012;36(1 Suppl):106S-17S.

(c) (i) This work is licensed under a Creative Commons Attribution 4.0 International License. The images or other third party material in this article are included in the article's Creative Commons license, unless indicated otherwise in the credit line; if the material is not included under the Creative Commons license, users will need to obtain permission from the license holder to reproduce the material. To view a copy of this license, visit http://creativecommons.org/licenses/by/4.0/

(c) The Author(s) (2017) 